livraisons

d'Histoire

de l'Architecture

\section{Livraisons de l'histoire de l'architecture}

34 | 2017

Le bois

\title{
Une postérité pour Nicolas Fourneau, maître- charpentier et savant ouvrier
}

"A posterity for Nicolas Fourneau, master carpenter and worker scientist"

„Ein ,Nachleben' für Nicolas Fourneau, Zimmermeister und gelehrter

Handwerker"

\section{François Calame}

\section{(2) OpenEdition}

\section{Journals}

Édition électronique

URL : http://journals.openedition.org/lha/798

DOI : 10.4000//ha.798

ISSN : 1960-5994

Éditeur

Association Livraisons d'histoire de l'architecture - LHA

Édition imprimée

Date de publication : 15 décembre 2017

Pagination : 17-26

ISSN : $1627-4970$

\section{Référence électronique}

François Calame, "Une postérité pour Nicolas Fourneau, maître-charpentier et savant ouvrier »,

Livraisons de l'histoire de l'architecture [En ligne], 34 | 2017, mis en ligne le 15 décembre 2019, consulté le 22 mars 2020. URL : http://journals.openedition.org//ha/798 ; DOI : https://doi.org/10.4000//ha.798 
Par François CALAME

\section{UNE POSTÉRITÉ POUR NICOLAS FOURNEAU, MAÎTRE CHARPENTIER ET SAVANT OUVRIER}

Nicolas Fourneau (1726-1792) est une figure très discrète du siècle des Lumières et un savant atypique de par son appartenance au monde ouvrier. Il n'en a pas moins joué un rôle essentiel dans la transmission des savoirs pour le métier de charpentier. Mais bien au-delà, son œuvre capitale, L'Art du Trait de charpenterie ${ }^{1}$, a fait passer un savoir oral populaire au domaine de l'art savant. Cette publication par un homme de métier fut une grande première, saluée par les encyclopédistes et l'Académie des sciences de Rouen. À ce titre, on peut considérer que Fourneau a été aux prémices de la reconnaissance par l'UNESCO de l'Art français du tracé de charpente.

Reconnu depuis 2009 au titre du patrimoine culturel immatériel de l'humanité, l'art français du Trait de charpente figure aujourd'hui dans une liste, un peu surréaliste, qui allie le repas gastronomique des Français, l'école française d'équitation de Saumur et le chant polyphonique corse. Il représente une petite partie de ce que les Français ont développé au cours de leur histoire, en matière de patrimoine. Il est à la fois subtil parce qu'immatériel, mais fort par l'importance du message spirituel, technique et social qu'il revêt. Il comporte un lien étroit avec le compagnonnage, ce système français de société ouvrière fermée ${ }^{2}$ instaurant l'obligation du voyage comme principe d'apprentissage. Et pourtant le Trait a été également pratiqué par d'autres professionnels que les compagnons. L'apport de Fourneau se place justement à l'intersection entre le monde très réservé des compagnons et celui des charpentiers non affiliés au Tour de France. Puisque l'on commémore cette année les deux cent cinquante ans de l'œuvre de Fourneau, l'occasion était forte de présenter quelques éléments en partie inédits sur ce novateur de génie. Un des aspects de son travail éclaire le rapport particulier qui existe entre praticiens du bâtiment et savants de tradition universitaire. On découvrira notamment que la pratique du Trait de charpente ne doit rien à l'approche pourtant révolutionnaire de la géométrie descriptive introduite par Gaspard Monge à la fin du XVIII ${ }^{\mathrm{e}}$ siècle.

On sait par les simples observations des voyageurs que l'art de construire des différents peuples admet de multiples usages, et ce qui est valorisé chez les uns peut sembler dérisoire chez les autres. En matière de charpente en bois, il existe des manières très diverses d'utiliser la matière. Malgré des règles physiques apparemment universelles et incontournables, les manières de bâtir sont fortement influencées par

1. Nicolas Fourneau, L'Art du trait de charpenterie, Rouen, Laurent Dumesnil, 1767, 125 p.

2. Le compagnon Stéphane Goubet préfere dire que sa société est "un peu secrète, ou plutôt discrète ". 
des représentations mentales et sociales. Amos Rapoport dans son Anthropologie de la maison ${ }^{3}$, nous l'a démontré : la technique et la matière proposent, mais ce sont les hommes qui disposent.

Si de nombreux bâtisseurs en bois ont résolu depuis longtemps par des moyens simples l'art de construire des bâtiments plus ou moins complexes, les charpentiers français ont très tôt, dès le Moyen Âge, commencé à introduire des subtilités croissantes dans la conception en volume de leurs ouvrages, tout au moins pour l'architecture savante. La géométrie plane est maîtrisée au $\mathrm{XI}^{\mathrm{e}}$ siècle dans la tradition de l'architecture romane mais l'apparition de la géométrie qui appartient réellement au domaine du Trait ne se perçoit que dans les raccords (arêtiers et noues) et il faut attendre le XIII ${ }^{\text {e }}$ siècle pour voir de significatives avancées dans le domaine du Trait de charpente.

Ainsi l'examen qu'a fait le compagnon Marcel Le Port de la charpente de la cathédrale d'Auxerre bâtie pour son abside dans la première moitié du XIII ${ }^{e}$ siècle met en évidence la maîtrise d'un tracé par le rabattement des arêtiers et le tracé très précis des coupes d'empanons ${ }^{4}$. La maîtrise de ces tracés est d'autant plus notable que par ailleurs toutes les cotes et les distances relevées dans l'édifice sont irrégulières, interdisant toute reproduction standard du travail, d'un arêtier à un autre. Le résultat, encore observable aujourd'hui, donne des longueurs de bois extrêmement justes et adaptées à la morphologie de la maçonnerie, et surtout des coupes biaises dans les deux sens parfaitement maîtrisées pour les empanons, sans les approximations que l'on observe dans des travaux obtenus de manière empirique.

Il faut préciser ici que dans les manières de bâtir à travers le monde, et dans l'art de construire en bois, il se pratique fréquemment des procédés que, en français, on appelle "par perchage ", c'est-à-dire des procédés pratiques où le dessin et le tracé n'ont pas leur place, ou plus exactement ne sont pas nécessaires. Dans la manière d'obtenir l'arêtier des croupes, très courante dans toute l'Europe centrale, les charpentiers procèdent de cette manière empirique, en évaluant la longueur nécessaire de bois par une perche ou une ficelle présentée en vraie grandeur sur le chantier. Le résultat fonctionne parfaitement mais tolère une approximation dans la perfection de l'exécution et souvent un tâtonnement un peu fastidieux dans les manœuvres.

Les Français, gens plutôt impatients et souvent attirés par un désir de rationalité, ou une certaine cérébralité dans leur rapport à la réalité, ont été attirés très tôt par le besoin de maîtriser, à la racine même de la démarche de construire, les procédés de traçage de leurs pièces de bois. Ce qui a motivé les constructeurs en bois c'est de déterminer en vraie grandeur les dimensions des pièces qui échappent à la géométrie plane. Sur l'épure d'une ferme, par exemple, l'élévation permet d'indiquer sans le moindre mystère les dimensions et les coupes des pièces de bois : arbalétrier, contrefiche, entrait, etc. Mais dès que les pièces de charpente obliques interviennent

3. Amos Rapoport, House form and culture, Englwood Cliffs, N. J., Prentice Hall., Inc., 1969, 150 p.

4. Marcel Le Port, "Historique du Trait, Le trait de charpente et ses applications ", Ephrem Longépé, et al. (Eds), Encyclopédie des métiers, la charpente et la construction en bois, Paris, Librairie du compagnonnage, 1981, t. 3, Le Savoir du Charpentier, fascicule 7, p. 283. 
à l'intersection de deux plans, elles échappent à l'élévation ainsi qu'à la vue en plan. C'est alors qu'intervient le procédé du rabattement ou " mise en herse ", dont on sait qu'il est maîtrisé en France dès le début du XIII ${ }^{\mathrm{e}}$ siècle pour les édifices majeurs, tandis qu'à la campagne certains constructeurs dans le cadre de l'architecture vernaculaire, remplacent le tracé par un procédé empirique jusqu'au début du $\mathrm{XX}^{\mathrm{e}}$ siècle.

Tout au long du Moyen Âge et au cours des siècles suivants se perfectionne l'art du Trait avec la maitrise progressive de détails techniques de plus en plus ambitieux. Ces procédés en évolution constante sont aujourd'hui perceptibles uniquement par les professionnels de haut niveau. Ce sont des innovations par les charpentiers et pour les charpentiers. Leur finalité peut consister à simplifier ou rendre plus rationnelle l'exécution de ce qui semble être de simples détails pour le non-charpentier. C'est le cas par exemple de la réalisation des arêtiers de section pentagonale dont le compagnon Le Port note l'apparition à partir du début du $\mathrm{XV}^{\mathrm{e}}$ siècle. La maîtrise de ce point permettait ainsi aux charpentiers d'obtenir des coupes "tournisses" pour tous leurs empanons, c'est-à-dire des coupes d'équerre au moins dans un plan. La simplification par rapport à des pièces obliques dans les deux plans est évidente. On citera en exemple l'observation faite par le compagnon Stéphane Goubet sur la tour carrée du Saint-Esprit de Besançon (Franche-Comté), datée de 1460 et qui présente de magnifiques arêtiers de vingt-deux mètres de long à section pentagonale parfaitement maîtrisée 5 .

Mais dans d'autres cas, note toujours Le Port, les exigences techniques raffinées correspondent au contraire au souhait de complexifier le travail, ce qui a de quoi nous étonner aujourd'hui. Quelle justification trouver à cela pour l'homme moderne, avide de simplification? Certaines complications sont liées à un souci esthétique perceptible par tous les observateurs, professionnels ou profanes. C'est le cas par exemple de voûtes cintrées, lambrissées ou non, dont l'aspect évoque celui des coques de bateau renversées. C'est par ce nom métaphorique d'ailleurs que le grand public désigne ces charpentes en France, croyant du même coup, à tort, que ces charpentes sont l'œuvre de charpentiers de marine.

Plus surprenantes sont les complications techniques qui resteront irrémédiablement illisibles par le public. On y trouvera ici les multiples barbes et chaperons que le charpentier digne de ce nom met un point d'honneur à réaliser sur les assemblages, pour compenser la matière manquante d'un côté par son équivalent sur la pièce complémentaire. Clairement, dans ces détails comme dans bien d'autres, le professionnel respecte les canons d'une esthétique purement interne à son corps de métier. Ce respect lui est précieux pour attester, à ses yeux comme à ceux de n'importe quel observateur averti, que celui qui a taillé cet ouvrage est un charpentier. Le vrai charpentier assortit par ailleurs systématiquement sa pratique d'une justification purement technique, minimisant la valeur emblématique de son geste.

Les perfectionnements peuvent par ailleurs sembler purement gratuits. Lorsqu'ils sont apparemment sans objet, ces perfectionnements sont très probablement à des

5. Recherches de Stéphane Goubet, 2014. 


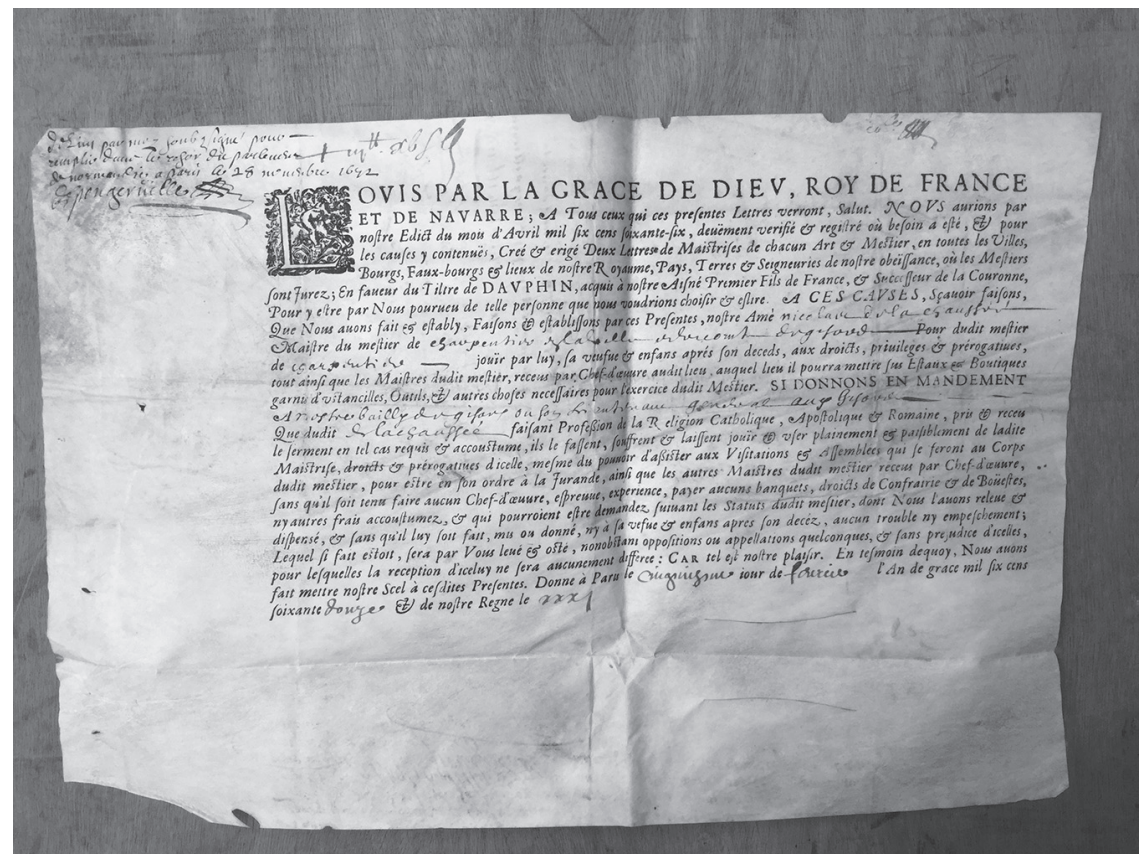

Ill. 1 : «Brevet de maîtrise d'un charpentier normand de 1672 ", Nicolas Delachaussée, autorisé à exercer son métier à Gisors et Étrépagny, collection particulière. (C) D. R.

fins pédagogiques ou initiatiques. "Certaines charpentes contiennent des difficultés apparemment inutiles " dit le Compagnon Le Port. Il faut souligner que le groupe social des charpentiers est fort structuré et comporte un apprentissage très réel du métier par la progression professionnelle, mais aussi par des mises à l'épreuve morales, dans laquelle la maîtrise de difficultés techniques, nécessaire ou imposée par le groupe, est primordiale.

On examinera en illustration ce brevet de maîtrise d'un charpentier normand de 1672 Nicolas Delachaussée, de Gisors, qui fait état de sa qualité de "maître du métier reçu par chef-d'œuvre» (ill. 1). Il est fait allusion dans ce document aux nombreuses pratiques décrites dans les "statuts " du corps de métier à cette époque, destinées à établir la qualité de membre autorisé de cette société. On y lit notamment l'existence de "chef-d'œuvre, épreuves et expériences » auxquels doivent satisfaire ceux qui veulent intégrer le métier.

Le métier de charpentier, qu'il soit lié ou non aux anciennes corporations, ou aux sociétés de compagnons, passées ou actuelles, présente un caractère initiatique fort. La pratique du Trait y joue un rôle très important ${ }^{6}$. L'apprentissage du Trait se fait par la tradition orale et la démonstration, dans les ateliers et sur les chantiers.

6. Voir dans ce même volume, la contribution de Nicolas Adell, «Les savoirs des "Bois debout" : le trait et l'Orient". 
Peu de documents en témoignent car les sources écrites sont absentes, et les innombrables exercices de Trait et travaux d'élèves ont disparu. À notre connaissance, un seul travail d'apprenti charpentier a été identifié, gravé en 1762 à la pointe sèche et au compas, sur un sol en plâtre sous le toit du palais de l'archevêque de Rouen. On voit bien qu'il s'agit d'un travail d'étude car la charpente réelle qui la recouvre n'a rien à voir avec cet exercice. L'exercice représenté concerne un arêtier sur ferme biaise avec une représentation très ramassée dite "à la tourangelle ", pur exercice pratique du charpentier. Encore aujourd'hui, les jeunes charpentiers s'exercent sur leur temps libre à des parties de trait, sorte de gymnastique tant géométrique que pratique, puisque liée à la réalisation de constructions qui pourraient être tout à fait réelles ${ }^{7}$.

Penchons-nous à présent sur l'itinéraire singulier de Nicolas Fourneau, documenté grâce aux recherches de Valérie Nègre ${ }^{8}$. Originaire de Pontoise, il est fils d'un maître charpentier. Il prétend être resté illettré jusqu'à l'âge de vingt-huit ans. Ouvrier pâtissier à l'âge de dix ans, charpentier à treize ans, nous dit son seul biographe et ancien élève, Jean Henri Hassenfratz, il apprend le métier auprès des compagnons du Devoir et devient l'un d'eux. Aucun détail n'éclaire les rapports professionnels entretenus avec son père. Quoiqu'il en soit Fourneau enseigne bel et bien chez les compagnons à Paris. Il a toujours à cœur dans sa pratique de "démonstrateur de Trait " d'associer tous les publics, qu'ils soient compagnons ou non.

Mais avant de devenir enseignant, Fourneau exerce longuement le métier en Normandie et parvient au titre de maître charpentier vers 1750, à l'âge de vingthuit ans, et donc, si l'on en croit Hassenfratz, sans savoir lire et écrire. Cette performance est à souligner, elle met en lumière le savoir très particulier d'un charpentier pourtant rompu aux exercices de géométrie complexe et de Trait. L'obtention du titre de maître, accordée par le roi, exige également une contribution financière sur laquelle nous ne savons rien. Fourneau décide alors de se vouer à la transmission du savoir du Trait, ainsi que ses propres expériences, notamment en matière de levage. Il prouve ainsi sa compétence étendue, autant dans le travail de conception graphique d'ouvrages complexes que dans la résolution des problèmes très concrets de chantier.

Fourneau signe son œuvre majeure en 1767 avec son traité L'Art du Trait de charpenterie, publié à Rouen. D'un ordre logique assez mystérieux et probablement inspiré de la méthode initiatique des compagnons, son traité constitue une première en France. Pourtant il reste à expliciter aujourd'hui quel rapport Fourneau a pu avoir avec la tradition allemande du Trait de charpente, très élaborée également à cette époque. On peut s'en faire une idée au moins à partir de l'œuvre de Johann Jakob Schübler, Ars Tignariae, publiée en 1735 et qui a visiblement de nombreux points de référence relatifs à l'art français du Trait de charpente.

7. François Calame et Andy Hyde, «Le trait de charpente, un art populaire à la française », F. Calame \& A. O. Martinussen, Charpentiers sans frontières, l'atelier de Normandie, Rouen-Caen : Les carnets d'ici - DRAC Haute-Normandie, 2013, p. 134-141.

8. Valérie Nègre, L'Art et la matière, les artisans, les architectes et la technique (1770-1830), Paris, Classiques Garnier, 2016, 324 p. 


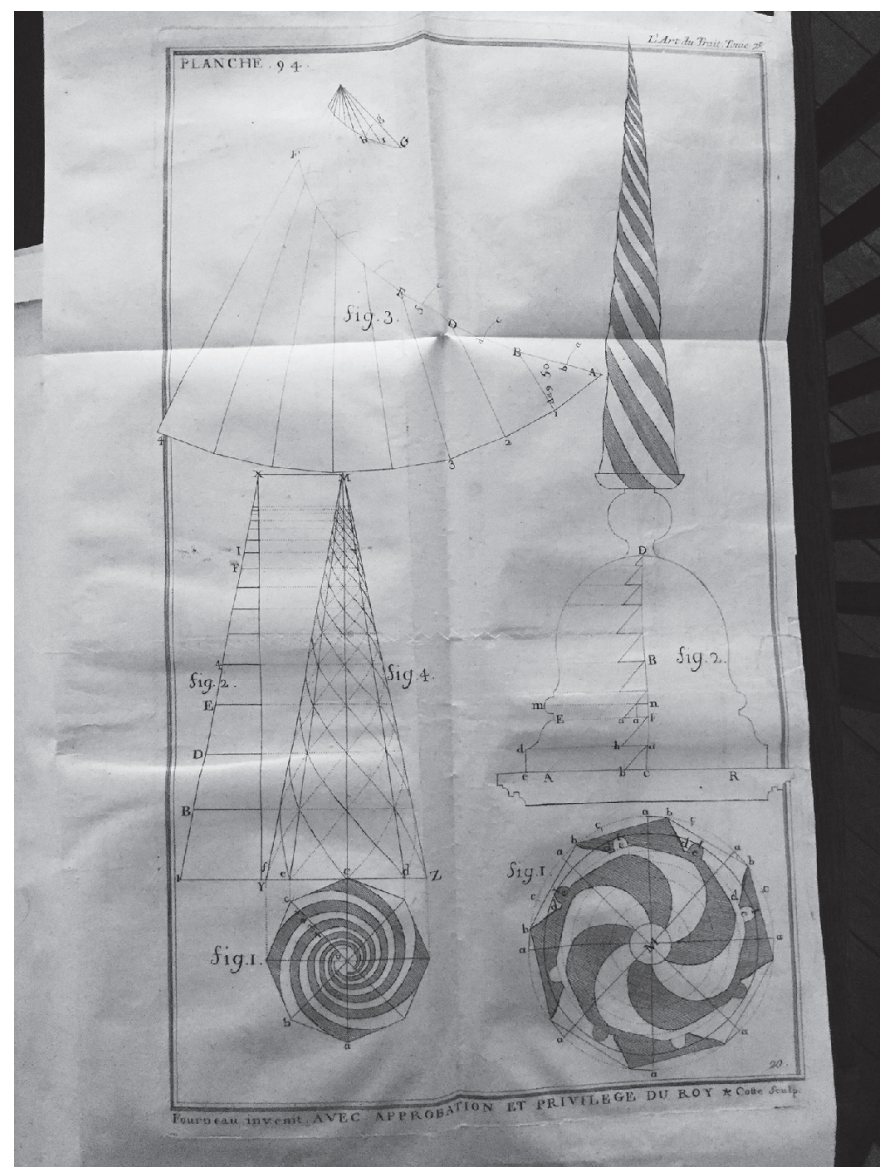

Ill. 2 : "Plan et élévation de la flèche torse de la Chartreuse de Bourbon-lez-Gaillon, plans simplifiés de Nicolas Fourneau ", publié dans son livre L'art du Trait de charpenterie, et qui a servi de base de travail aux deux aspirants taillant leur réception à Rouen à l'hiver 2016-17, collection particulière. (C D. R.

Fourneau, on l'a vu, est tout à la fois un pédagogue et un communiquant. Mais avant d'être tout cela, il a été un impressionnant homme de chantier, rompu à tous les aspects du métier depuis les tracés jusqu’à la réalisation et au levage. Il convient en particulier de signaler deux de ses réalisations majeures. L'une concerne les premiers exemples construits en France de lucarne en guitarde, dont deux spécimens ornent à Rouen l'hôtel du Président de Bailleul vers 1764. Premiers exemples attestés et modèle du genre, ces guitardes deviendront un archétype décliné en variantes multiples tout au long du XIX ${ }^{\mathrm{e}}$ siècle et encore volontiers actualisé grâce au lamellécollé chez les compagnons du Tour de France, lors des travaux de réception des aspirants. Le deuxième exemple, aujourd'hui malheureusement disparu, fut la construction de la première flèche torse en charpente qui couronnait la chartreuse de Bourbon-lez-Gaillon (Eure) en 1767 (ill. 2). Cet ouvrage fut entièrement conçu 


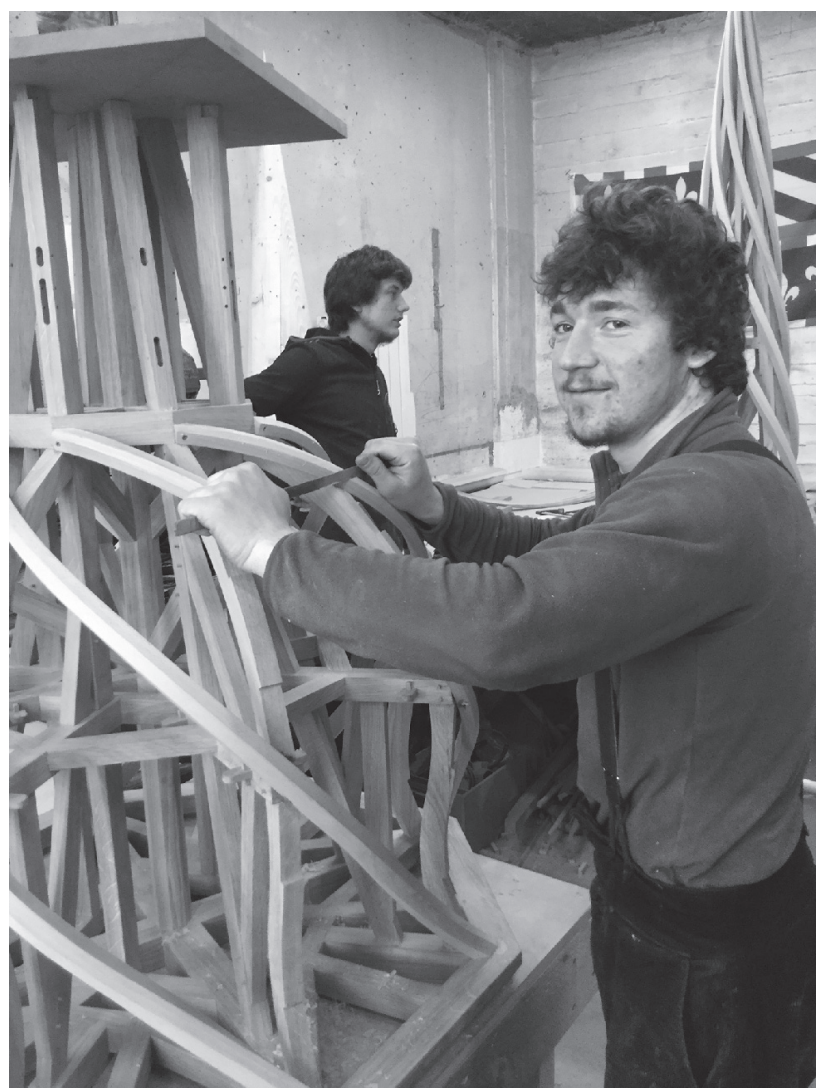

Ill. 3: "Dans leur "renardière", les deux aspirants charpentiers Tino Piaia et Bastien Bourotte, de l'AOCDTF, travaillent jour et nuit à la réalisation de leur chef-d'œuvre ", hiver 2016-17. C C Cl. François Calame.

par Fourneau à partir des plans très simples, pour ne pas dire assez quelconques, de l'architecte parisien Hélin. La flèche d'une hauteur finale de 11 mètres se composait d'un dôme tors surmonté d'une boule, elle-même coiffée d'une flèche torse. Cette réalisation est représentée de façon assez schématique dans le traité de Nicolas Fourneau.

L'année 2017 représentant une occasion particulière de saluer l'œuvre de Nicolas Fourneau, dans la perspective de la liste de l'UNESCO, j'ai proposé en 2016 à la corporation des Charpentiers de l'Association Ouvrière des Compagnons Charpentiers du Devoir du Tour de France que l'ouvrage de la flèche torse de Bourbon-lez-Gaillon puisse constituer un sujet d'inspiration pour un aspirant taillant son travail de réception. En fait, ce sont deux jeunes charpentiers qui ont uni leurs efforts dans ce sens. Il faut nommer ici Tino Piaia et Bastien Bourotte, deux aspirants de vingt et un et vingt-deux ans qui ont consacré quatre mois, sur leurs soirées et leurs congés, pour tracer et tailler ce chef-d'œuvre d'après les épures de Fourneau (ill. 3). Cette 


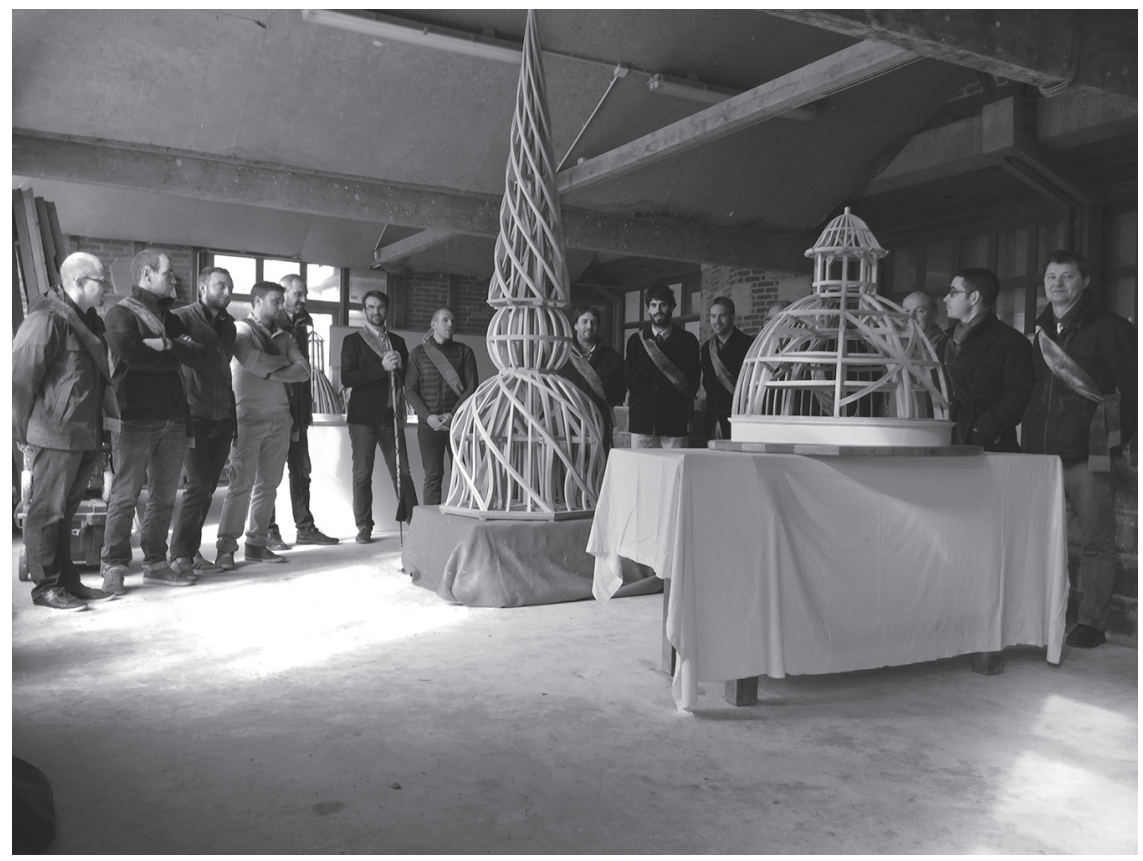

Ill. 4 : "La cayenne des Compagnons charpentiers du Devoir de Rouen se réunit pour examiner et corriger les travaux de réception préparés durant l'hiver par les "renards", et débouchant sur la cérémonie de la réception lors de la Saint-Joseph ", mars 2017. (C) Cl. François Calame.

maquette en chêne et hêtre massifs reproduit, moyennant certaines suppositions et interprétations, la réalité très concrète de ce que devait être cet ouvrage unique en son temps (ill. 4).

Ce chef-d'œuvre est aujourd'hui exposé au Musée d'Art, d'Histoire et d'Archéologie d'Évreux, et a été présenté pour la première fois au public lors de l'exposition "Une Renaissance en Normandie ", consacrée à l'œuvre du Cardinal Georges d'Amboise et à son Palais de Gaillon (juillet-octobre 2017).

Encore aujourd'hui, chez les compagnons charpentiers circule volontiers l'adage "l'Orient vaut le Trait». Cette phrase non compréhensible par le profane proclame que le métier de charpentier se nourrit autant de l'esprit pratique et de la débrouillardise, que de l'esprit subtil et plus cérébral de la pratique du Trait. Gaspard Monge théorise sa géométrie descriptive selon des procédés parallèles et bien distincts ${ }^{9}$. Il est probable que Fourneau et Monge se sont croisés, probablement fréquentés. Il est aujourd'hui admis que la géométrie descriptive, savoir académique constitué comme une discipline universelle, doit plus au savoir ancestral de Trait et de stéréo-

9. Yves Deforge, Le Graphisme technique : son histoire et son enseignement, Paris, Champ Vallon, 1981, $255 \mathrm{p}$. 
tomie des gens de métier, que l'inverse. S'il est incontestable que le savoir de l'ingénieur fait un bond considérable grâce aux apports de Gaspard Monge, et ce à destination de l'ensemble des domaines d'activité et de production, il convient de rétablir à leur juste place les apports patients et progressifs des bâtisseurs européens, en particulier charpentiers, mais bien sûr également tailleurs de pierre.

François CALAME

docteur en ethnologie (EHESS) conseiller pour l'ethnologie à la Direction régionale des affaires culturelles de Normandie 
